\title{
Raman Spectroscopy of the Aguas Zarcas CM2 Meteorite
}

\author{
Xenia Ritter, Katarina Keating, Xin Yang, Jennika Greer and Philipp R. Heck
}

Robert A. Pritzker Center for Meteoritics and Polar Studies, Negaunee Integrative Research Center, The Field Museum of Natural History, Chicago, Illinois, United States

The starting materials, processes, and conditions $~ 4.6$ billion years ago defined the composition of the present-day Solar System. Carbonaceous chondrite meteorites can preserve an almost unaltered record of these starting materials. This includes minerals and organics inherited from the interstellar medium in the presolar molecular cloud and younger components from the protoplanetary disk before planets formed. Studying these materials can provide important insight into the compositions, processes, and chemical conditions of the early Solar System. This not only helps us better understand the early determining stage of the Solar System evolution, but also enables us to investigate parent body processes such as thermal and aqueous alteration of materials. In this study, we investigate the thermal alteration of the Aquas Zarcas meteorite.

The Aguas Zarcas CM2 chondrite is a recent meteorite fall that has undergone minimal amounts of parent body aqueous and thermal alteration. It fell on April 23, 2019 in Costa Rica and was collected rapidly after the fall and before a rain event. The meteorite is a breccia with a main lithology similar to the Murchison CM2 chondrite and similarly rich in organic matter [1-7]. Other lithologies include matrix-rich rock fragments with almost no chondrules and a distinct lithology exceptionally rich in meteoritic iron [8]. Another lithology resembles the texture in the Paris CM chondrite and, like Paris, contains primitive GEMS-like objects [9]. Due to the unusual diversity of petrological textures it is desirable to investigate the different degrees of alteration in each of the lithologies present. Recent studies focused on aqueous alteration [1-3, 6-7, 9]; in this study we focus on the different degrees of thermal alteration these different lithologies experienced.

Systematic studies with Raman spectroscopy of the organic matter in the matrix of unequilibrated meteorites, including carbonaceous chondrites and unequilibrated ordinary chondrites, have revealed trends of organic matter D (disordered, $1355 \mathrm{~cm}-1$ ) and $\mathrm{G}$ (graphite, $\sim 1581 \mathrm{~cm}-1$ ) peak parameters with increasing thermal alteration [11-13].

We have analyzed two polished sections containing 14 fragments of Aguas Zarcas (FMNH ME 6111.20 and FMNH ME 6111.21) comprising at least three different lithologies. The mounts include at least one representative sample of each lithology that has been found to date. We have used a WITec alpha300 R Raman spectroscopy system at the Field Museum equipped with a $532 \mathrm{~nm} \mathrm{Nd:YAG} \mathrm{laser,} \mathrm{operating} \mathrm{at}$ $0.4 \mathrm{~mW}$, either 600 or $1800 \mathrm{~g} / \mathrm{mm}$ grating and 10x or 50x magnification lenses. Integration and accumulation time ranged between 0.6-1 s and 40-200 s, respectively. A total of 180 Raman spectra were measured on Aguas Zarcas, with 5-30 (on average 13) spectra on each fragment. Murchison (CM2), Allende (CV3), Tieschitz (H/L.3.6), Colony (CO3.0) and Mighei (CM2) were measured as reference samples. All Raman spectra were corrected for cosmic ray spikes prior to spectral processing in Fityk [14]. A linear background was first subtracted followed by fitting the D and G peaks to a Lorentzian function.

Our Raman analysis places Aguas Zarcas in the same parameter range as the Murchison CM2 chondrite (Fig. 1). The variations of the widths of the $\mathrm{D}$ and $\mathrm{G}$ peaks as well as the D/G peak intensity ratios did not vary less within the individual fragments than they did among the different fragments (Fig. 2). This implies 
that the degree of thermal alteration experienced by the organic matter in the different lithologies was strikingly similar. The different petrological texture is therefore likely the result of different degrees of aqueous alteration rather than thermal. This conclusion is consistent with the conclusions drawn independently by studies using different methods [e.g., 1].

Acknowledgments: We thank Terry Boudreaux and the Boudreaux family for donating the Aguas Zarcas meteorite to the Field Museum. We acknowledge the TAWANI Foundation for providing a major grant for funding of the Robert A. Pritzker Center for Meteoritics and Polar Studies. PRH thanks an anonymous donor for establishing the Field Museum's Science Innovation Award 2020 which funds part of this work.

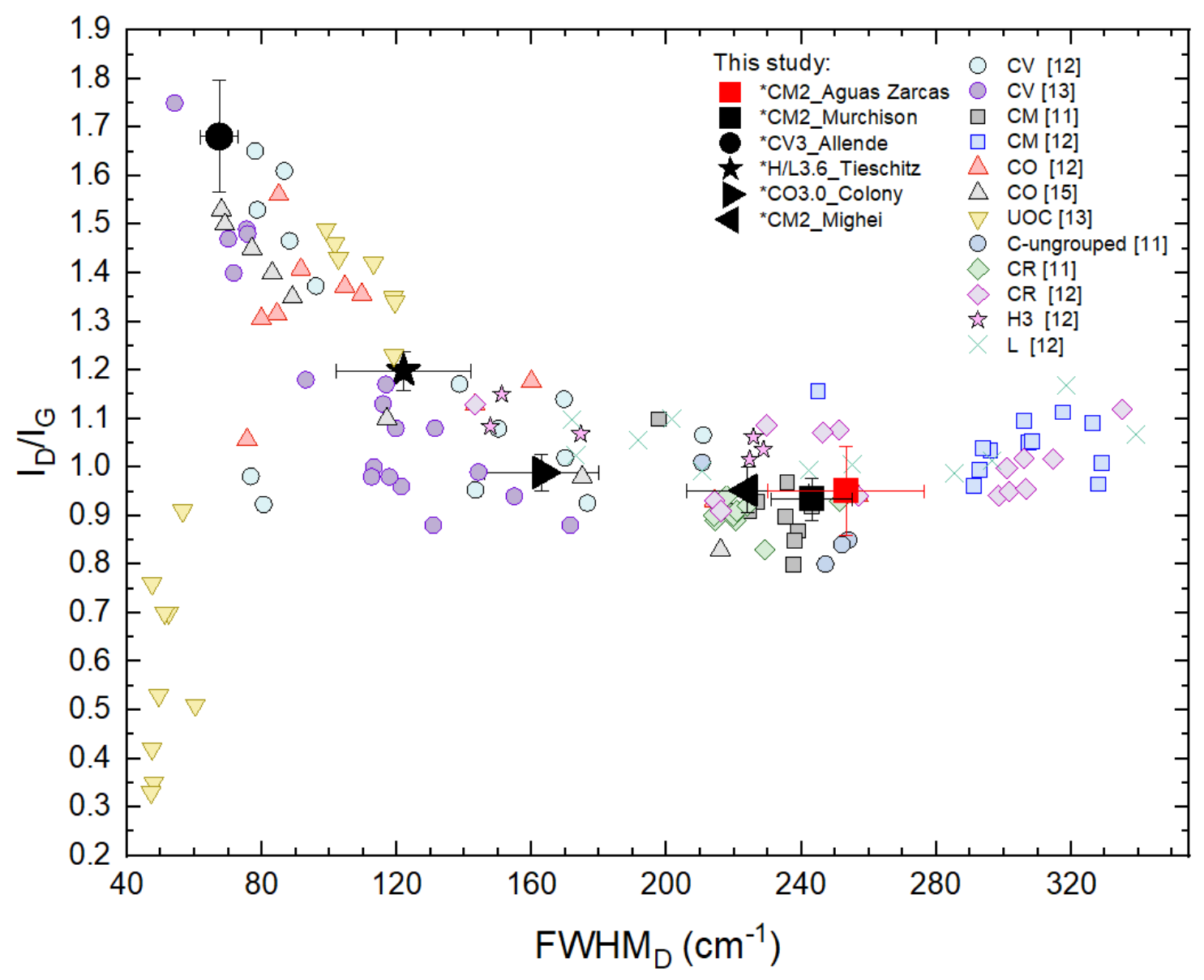

Figure 1. The intensity ratio ID/IG as a function of FWHMD is plotted using selected literature data [11$13 ; 15]$ and data from this study (black and red symbols; error bars display standard deviation). Aguas Zarcas (in red) is identical to the Murchison CM2 chondrite within uncertainties. *This study. 


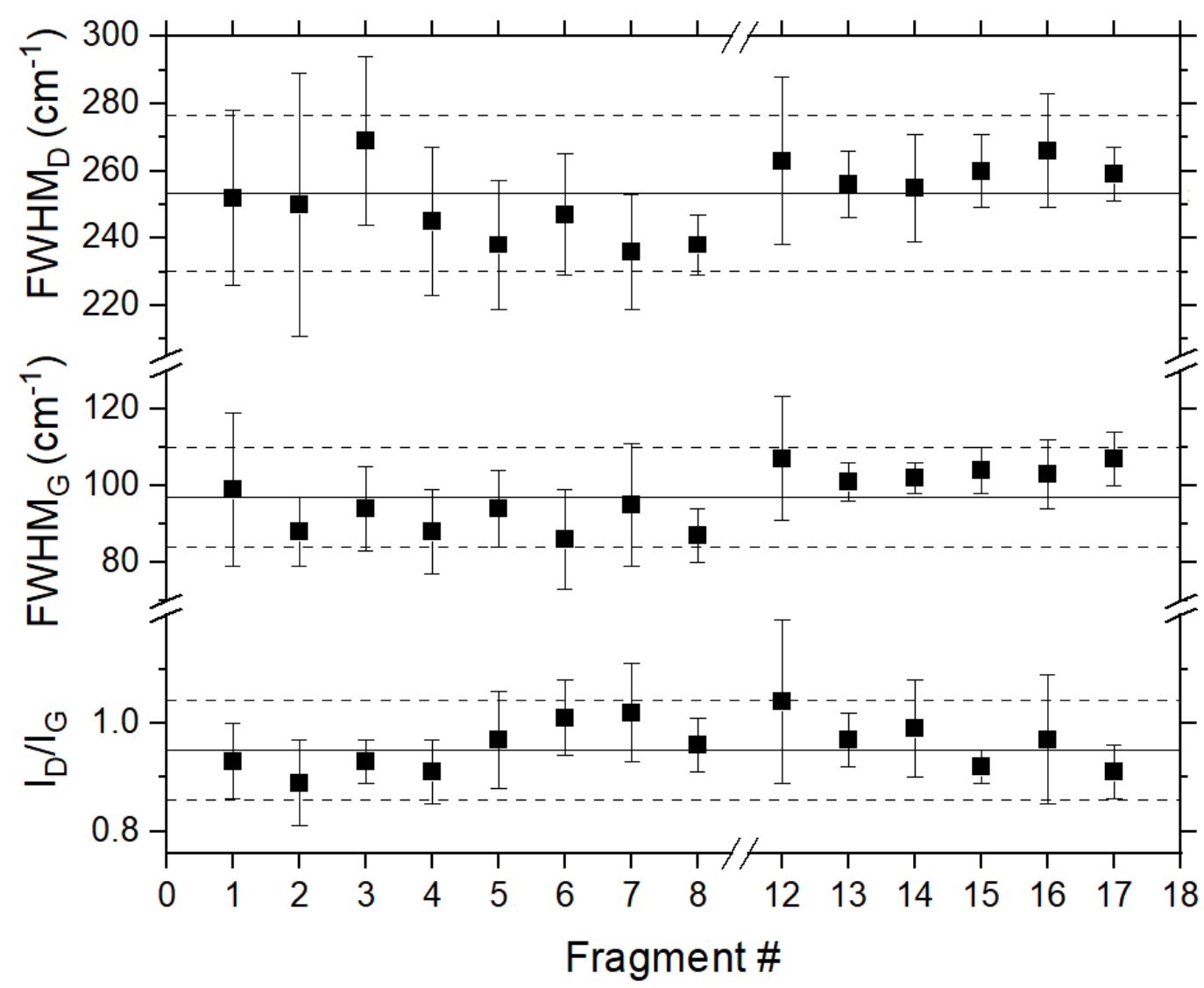

Figure 2. Statistical summary of all measured Raman spectra on Agua Zarcas in this study, showing the average (solid line) and standard deviation (dashed line) for ID/IG, FWHMG and FWHMD for each fragment. Note that fragments 9, 10 and 11 were dismissed during post processing due to small number of analyses. Error bars are $+/-1$ standard deviation.

References

[1] J Davidson et al., LPSC (2020), \#1623.

[2] I Kerraouch et al., LPSC (2020), \#2011.

[3] L J Hicks and J C Bridges, LPSC (2020), \#2869.

[4] D P Glavin et al., LPSC (2020), \#1025.

[5] L D Tunney et al., LPSC (2020), \#1795.

[6] I Kouvatsis and J A Cartwright (2020), \#2512.

[7] R Findlay et al., LPSC (2020), \#2880.

[8] A Bouvier, et al., The Meteoritical Bulletin, 108 (2019).

[9] K L Villalon et al., LPSC (2020), \#2757.

[10] P M C Martin and M R Lee, LPSC (2020), \#1375.

[11] E Quirico et al., GCA 136 (2014), p. 80-99.

[12] H Busemann, C.M. Alexander and L Nittler, Met. Planet. Sci. 42 (2007), p. 1387-1416.

[13] L Bonal et al., GCA 189 (2016), p. 312-337.

[14] M Wojdyr, J. Appl. Cryst. 43 (2010), p. 1126-1128.

[15] L Bonal et al., GCA 71 (2007), p. 1605-1623. 